\title{
Nitrogen status assessment for multiple cultivars of strawberries using portable NIR spectrometers combined with cultivar recognition and multivariate analysis
}

\section{Gangshan Wu}

Jiangsu Vocational College of Agriculture and Forestry

\section{Qiyou Jiang}

Jiangsu Vocational College of Agriculture and Forestry

\section{Yuhao Bai}

Nanjing Agricultural University

Chongfeng Tian

Jiangsu Vocational College of Agriculture and Forestry

Wei Pan

Jiangsu Vocational College of Agriculture and Forestry

Baohua Zhang ( $\sim$ bhzhang@njau.edu.cn)

Nanjing Agricultural University https://orcid.org/0000-0002-7731-2518

\section{Research}

Keywords: FT NIR spectroscopy, Cultivar variation, Cultivar models, SPAD value, N fertilizer 41 management

Posted Date: December 20th, 2019

DOI: https://doi.org/10.21203/rs.2.19371/v1

License: (c) (i) This work is licensed under a Creative Commons Attribution 4.0 International License. Read Full License 
Nitrogen status assessment for multiple cultivars of strawberries using portable

NIR spectrometers combined with cultivar recognition and multivariate analysis

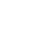

$$
\text { Gangshan Wu', Qiyou Jianga, Yuhao Bai }{ }^{\mathrm{b}} \text {, Chongfeng Tianª Wei Panª , Baohua }
$$

(1)

$$
\begin{gathered}
\text { Zhang }^{\mathrm{b}^{*}} \\
{ }^{\mathrm{a} C o l l e g e} \text { of Information Engineering, Jiangsu Vocational College of Agriculture and } \\
\text { Forestry, Jurong, Jiangsu, PR China }
\end{gathered}
$$

${ }^{\mathrm{b}}$ College of Engineering, Nanjing Agricultural University, Nanjing, Jiangsu, PR China (1)

*Correspondence should be addressed to Baohua Zhang (bhzhang@njau.edu.cn). The primary contact's telephone number is $+86(025) 58606585$ and the fax number is $+86(025) 58606585$.

\section{ABSTRACT}

Background: The assessment of nitrogen status non-destructively in strawberry was performed to indicate its growth and provide guidance for precise management of $\mathrm{N}$ fertilizer using near-infrared reflectance (NIR) spectroscopy with leaf spectral reflectance. The Leaf soil plant analysis development (SPAD) value was thought as an indicator data that indirectly reflects nitrogen status in strawberry. However, the variation of cultivars would lead to differences in the cell structure and light scattering and/or reflection effects of strawberry leaves when assessed strawberry $\mathrm{N}$ status, which caused that no single leaf SPAD threshold value or same $\mathrm{N}$ demand can be 
applied for all strawberry cultivars. As a result, accurate detection of SPAD values and $\mathrm{N}$ status in strawberries with multiple cultivars is still challenging.

Results: In this study, Individual-cultivar model, hybrid-cultivar model and multi-cultivar model were developed for the determination of SPAD values, and the performance of the models in lessening the impact of cultivar variation were studied and compared. Individual-cultivar model was constructed on the basis of the single cultivar of strawberry leaf; hybrid-cultivar model was developed by merging the spectrum reflectance data and SPAD values of all studied leaf samples, and multi-cultivar model was built in combination with cultivar identification, individual-cultivar models, and model search strategy.

Conclusion: The results indicated that multi-cultivar model was more superior to the other two models for SPAD values estimation of strawberry leaves from different cultivars, with the overall Rp and RMSEP value respectively being 0.966 and 0.468 . We demonstrate that the leaf $\mathrm{N}$ content per strawberry is profoundly affected by cultivar variation, and establishing a multi-cultivar model might be more useful in monitoring nitrogen status and guiding $\mathrm{N}$ fertilization recommendations for different strawberry cultivars.

Keywords:

FT-NIR spectroscopy; Cultivar variation; Cultivar models; SPAD value; N fertilizer management

\section{Background}

Nitrogen $(\mathrm{N})$ is one of the most important nutrients for strawberry growth, which can 
increase chlorophyll content concentration, improve the photosynthetic efficiency and delay the aging [1]. A proper nitrogen fertilizer management could promote the strawberry marketable yields and improve the quality. Therefore, it is crucial to real-time monitor the dynamic $\mathrm{N}$ requirement of strawberry with diverse cultivars to achieve optimal $\mathrm{N}$ fertilizer management. Conventional physicochemical assessing methods of nitrogen status, such as Kjeldahl nitrogen detection method, tissue and chemical analysis, remote sensing with aerial images have been successfully employed in cultivar-specific crop production management. However, although most of them exhibit high accuracy and great reliability, they have some certain limitations, namely, time-consuming, costly and susceptible to the background noises and low resolution [2]. The application of non-destructive methods, such as NIR spectroscopy and chlorophyll meter SPAD instruments, to real-time estimate the nitrogen status on strawberry, has promise for improving $\mathrm{N}$ fertilizer managements during the growth and cultivation of strawberries.

There exists a very close relationship between nitrogen content and chlorophyll concentration in the leaf [3]. As the most important chelates for plants, chlorophyll is also an important indicator of plant nitrogen status and leaf senescence, is capable of responding to environmental stresses in real time [4]. SPAD value is the relative content of chlorophyll, which has a good correlation with the measured value of chlorophyll and nitrogen content. Therefore, SPAD value measurement offers an excellent strategy to reflect nitrogen status in plants and synchronize $\mathrm{N}$ supply with actual plants demand. 
In recent years, owing to its strong advantage in the observation and application of regional ecological parameters in large areas of vegetation, NIR spectroscopy technology has been widely used in the quantitative analysis of plant biochemical parameters $[5,6]$. In combination with multivariate calibration analysis such as principle component regression (PCR), Least squares support vector machine (LS-SVM), and stepwise multiple linear regression (SMLR), NIR spectroscopy technique has been successfully applied and developed for data mining and quantitative analysis in agricultural applications. Feng et al. developed a quantitative model for on-line monitoring of nitrogen status on wheat leaves using estimation indices and key NIR bands in wheat in 2008 [7]. Min et al. adopted NIR spectra coupled with SMLR method to detect the degree of nitrogen stress in Chinese cabbage leaves in 2006 , the result showed the high determination coefficient $\left(R_{p}\right)$ of 0.846 [8]. Moreover, the NIR technique has also been successfully used in classification research to confirm the capacity of NIR performance in detecting fruits from diverse cultivars [9, 10] and ripening stages [11-13].

Strawberry is a complicated natural fruit with a number of cultivars. Numerous studies have found that the chlorophyll content per leaf and $\mathrm{N}$ demand varied widely among different strawberry cultivars [14-16]. This variability is presumed to be due to variability of cellular structural differences among the leaves that cause different light scattering and/or reflection effects. Hence, no single leaf SPAD threshold value or same $\mathrm{N}$ demand can be applied for all strawberry cultivars. Bavec et al. analyzed the difference between chlorophyll content in different cultivars of winter wheat, and 
further predicted grain yield accurately in 2001 [17]. Xiong et al. researched the multiple leaf characteristics of crop genotypes and cultivars on the SPAD-based leaf nitrogen assessment in 2015, and the results shown that nitrogen content is genetically determined and differences among cultivars are expected [18]. Therefore, due to the difference in nitrogen requirements of different strawberry cultivars, the impact of cultivar variations on the NIR spectra should be considered carefully when applied for chlorophyll content estimation and nitrogen management in precision agriculture.

Nevertheless, the reports on decreasing the influence of cultivar variation on the assessment of leaf SPAD values and achieve optimal dynamic $\mathrm{N}$ fertilizer management of strawberries using FT-NIR reflectance spectroscopy are scarce. Hitherto, there are two main methods to determine the leaf SPAD values with multiple cultivars. One method was to construct the individual-cultivar model based on the single cultivar of strawberry leaf for achieving accurate SPAD values measurement of strawberry leaves with multiple cultivars [19, 20]. For the second method, the calibration model called hybrid-cultivar model was developed by merging the spectrum reflectance data and SPAD values of all studied samples [21, 22]. However, numerous researches have shown that the best performing model for any cultivar was ultimately the model calibrated on that cultivar, and the application of the calibrated model to other or different cultivars consistently would lead to the loss of performance to vary degrees $[23,24]$. Therefore, application of the individual-cultivar models and hybrid-cultivar model may not be conducive for obtaining satisfactory prediction accuracy of leaves SPAD values in different strawberry cultivars. In this 
study, in order to render the impact of cultivar variation a negligible interference for leaf SPAD values detection and nitrogen stress levels estimation on strawberry with multiple cultivars, a multi-cultivar prediction model was proposed and developed. The specific objectives are to: (1) analyze and compare characteristics and difference of leaf NIR spectrum reflectance and SPAD reference values following different strawberry cultivars; (2) compare and estimate the performance of individual-cultivar models and hybrid-cultivar model in SPAD values determination with multiple strawberry leaf cultivars; (3) recognize and identify cultivars of strawberry leaves samples using Random forests classifiers with extracted characteristic fingerprint spectrum as inputs; (4) establish the optimal multi-cultivar model combining cultivar recognition model, individual-cultivar models and model search strategy, and further analyze its performance in detecting SPAD values of strawberry leaves from different cultivars; and (5) asses the leaf chlorophyll and nitrogen content on strawberry according to the predicted leaf SPAD values; analyze the different dynamic $\mathrm{N}$ requirements in diverse cultivars of strawberries and improve $\mathrm{N}$ fertilizer managements in precision agriculture.

\section{Materials and methods}

\section{Leaf samples collection}

The strawberries of three different cultivars, named Hongyan (Hy), Ningyu (Ny), Taoxun (Tx) were used in this study. They were picked up in the Agricultural expo garden of Jiangsu, China, grown in the same geographical and climatic conditions. Healthy mature leaves were randomly collected from the strawberry canopy. After 
133

134

collection, the extracted sampled leaves were immediately processed to ensure that they retained water, and subsequently they were kept in dark and cool conditions to decrease the potential influence of light intensity on chloroplast movement [25]. A total of 300 samples of strawberry leaves free of visual drawbacks (including shrivel, decay, scars, cuts, etc.) from three cultivars (hundred samples * three cultivars) were obtained for measurement. According to the Kennard-Stone algorithm [26], 75 samples of each cultivar were selected and used to establish the calibration models and the remaining 25 samples to construct the set of prediction.

\section{Spectral reflectance acquisition}

After the leaves were collected in the laboratory, leaf reflectance was measured using a USB2000 spectrometer with charge coupled device (CCD) detector (Oceanoptics Inc., USA), a NIR fiber-optic probe, a tungsten halogen lamp (12V, 25W) and a leaf clip over the $350-2000 \mathrm{~nm}$. The detector covered the spectral wavelength range between 340 and $1030 \mathrm{~cm}^{-1}$, with a spectral resolution of $3.4 \mathrm{~nm}$ and a sampling interval of $1.4 \mathrm{~nm}$. Before spectra reflectance acquisition, the instrument was calibrated with the $99 \%$ reference whiteboard [27, 28]. For each leaf sample measurement, the scan was repeated 32 times at the same position and the mean spectral reflectance value was recorded as the raw reflectance of the leaf for subsequent analysis.

The reflectance spectrum of leaf sample is usually affected by uninformative biological variability, which can generate noises and baseline shifts within wavelength regions related to chlorophyll concentration. Therefore, spectrum reflectance data 
pretreatment has become an important part for chemometric modeling and multivariate analysis. In our study, a series of pretreatment approaches including multiple scatter correction (MSC), standard normal variation (SNV), Savitzky-Golay smoothing and Norris gap were employed on the spectral data. After that, the spectrum reflectance data that pretreated by Savitzky_Golay smoothing [29] and MSC [30] algorithms were used for the subsequent multivariate calibration, because they have shown better performance in model prediction than other pretreatments.

\section{Measurement of leaf SPAD}

After spectral acquisition, a SPAD-502 chlorophyll meter (relative value of 0-100, Minolta, Osaka, Japan) was performed to measure the chlorophyll concentration of the strawberry leaves at the same position as the leaf spectrum measurements. The SPAD value of each leaf was uniformly measured for 5 times, and the mean value was taken as the final value of nitrogen status reference concentration of this leaf sample.

\section{Characteristic fingerprint spectrum exploration}

Infrared spectral fingerprint region contains a very complicated series of absorptions due to all manner of bending vibrations within the molecule [31]. When there is a slight difference in the molecular structure of a substance, it will cause a significant change in spectral absorption. The infrared absorption peak of material molecules is especially abundant in this region, which is as dense as human fingerprints and has significant features. This characteristic fingerprint can be used for the classification and identification of similar objects. However, it will be very difficult to directly use the original infrared spectra for object identification when the analysis system is 
complex [32]. There are many wave points in the infrared spectrum of strawberry leaves, and the spatial dimension of the data set is too high, which contains a lot of redundant information. Therefore, dimensionality reduction and feature extraction should be carried out in the data space, so as to extract the characteristic fingerprint spectrum from the data space and magnify the spectral pattern differences between strawberry leaves with different cultivars, and thus realize cultivars classification and identification. In this study, the Random Frog algorithm was used for dimensionality reduction and characteristic fingerprint spectrum exploration.

Random Frog is a useful method to extract relevant and effective data information from the full spectrum by eliminating the uninformative variables. It can be modeled with a small number of variable iterations and subsequently the selection probability (SP) of each variable are calculated and output. The execution of Random Frog is as follows: (1) Initialize a variable subset $\mathrm{V}_{0}$ which randomly contains $\mathrm{Q}$ variables; generate a random number from the standard deviation $\theta Q$ and normal distribution with mean value; round the number to its nearest integer, denoted by $Q^{*}$; (2) generate a subset $\mathrm{V}^{*}$ of candidate variable which includes $\mathrm{Q}^{*}$ variables; accept $\mathrm{V}^{*}$ as $\mathrm{V}_{1}$ with a certain probability and replace $\mathrm{V}_{0}$ with $\mathrm{V}_{1}$; loop through the above process until $\mathrm{N}$ iterations are completed; (3) compute the SP of each variable; selecte variables (wavelengths) with higher SP than the cutoff value as characteristic fingerprint spectrum, while variables with lower SP than the cutoff were considered to be uninformative and eliminated [33]. The detailed procedures of Random Frog algorithm for selecting variables were illustrated in these literatures [34-36]. 


\section{Random forest for strawberry leaves cultivar recognition}

As mentioned above, characteristic fingerprint spectrum could be applied to identify the spectral characteristics of the leaves of different cultivar of strawberry due to its immutability and uniqueness [37]. In this study, we select Random forests (RF) classifiers for cultivars recognition of strawberry leaves samples, having characteristic fingerprint spectrum as inputs.

$\mathrm{RF}$ is an ensemble classifier which executes by constructing a multitude of decision trees during training and outputting the pattern of the classes (classification) of the individual trees [38]. Moreover, in order to use different bootstrap samples of the data to construct each tree, RF change the way classification trees are constructed. Unlike other methods based on boosting, RF method is a classifier added an additional layer of randomness to bagging, which is not sensitive to noise. For the standard trees, each node uses the best partition of all variables; while in the RF, each node is partitioned using the best in the prediction subset randomly selected for that node [39]. Compared to other single classifiers such as neural networks, support vector machine, and machines discriminant analysis, RF classifier turns out to shown more robust against overfitting and better classification performance.

\section{Chemometric models for SPAD values prediction (PLS)}

PLS regression analysis is an extensively used multivariate calibration method, which is widely employed in the NIR spectral analysis due to its tolerance to large numbers of variables and insensitivity to collinear variables [40]. The principle of PLS is to perform a bilinear model that projects original independent information onto a new 
221 latent space, where the maximum covariance between the variable matrix $\mathrm{X}$

222 (reflectance spectrum data) and the chemical attribute matrix Y (the SPAD values) is maximized [23]. The maximum information extracted from a large number of highly

224 correlated collinear primitive variables could be compressed into less than 20 latent

225 variables. Latent variables (LVs) can reduce random errors and noise in the original 226 data, and consider the variability of the original variables as much as possible. In this 227 study, PLS was applied to construct calibration models using the reflectance spectrum 228 data and SPAD values for assessment of nitrogen status in strawberry leaves with 229 different cultivars; 10-fold cross-validation was employed to avoid overfitting of 230 calibration sets and estimate model prediction performance. The optimal number of 231 LVs was determined by the lowest root mean square error of cross validation 232 (RMSECV).

\section{Optimal wavelengths extraction}

234 To eliminate non-informative spectral data, facilitate results interpretation, and 235 improve the calibration model performance for estimating SPAD values of strawberry 236 leaves, optimal wavelengths selection is an inevitable and crucial procedure in 237 multivariate analysis [37]. In this paper, successive projections algorithm (SPA) was 238 applied to extract optimal wavelength for cultivar calibration models development 239 with high predictive capacity.

240 SPA is a feed forward variables selection strategy to solve collinearity problems for 241 multivariate calibration, which can be applied to extract the optimal wavelengths with minimal redundancy in the full spectrum [41]. The principle of SPA is that it begins 
with one variable (wavelength), and subsequently merges another one in each iteration until a specified number of variables are reached [42]. SPA comprises three phases: (1) selecting the maximum projection vector on the orthogonal subspace and wavelength variables with minimum redundancy and collinearity; (2) determining the optimal wavelengths on the basis of the smallest RMSEV in the validation set of calibration model; (3) removing uninformative variables by a backward elimination procedure without significant loss of prediction performance [43]. Subsequently, an optimal subset of wavelengths extracted by SPA was used as the inputs for cultivar calibration models establishment and the performance of the models based on optimal wavelengths were studied.

\section{Flowchart of our proposed algorithm}

In order to decrease the impact of cultivar variation for leaf SPAD value detection and $\mathrm{N}$ fertilizer managements, a multi-cultivar prediction model was proposed and established in this study (Fig. 1).

\section{Fig. 1. Flowchart for prediction of SPAD values of strawberry leaves with different}

\section{cultivars using multi-cultivar model}

As shown in the above flowchart, the multi-cultivar model is essentially a combinatorial model with cultivar recognition model, and individual-cultivar models, and model search strategy. For the individual-cultivar models, they were established based on varying data (spectral data and SPAD value of strawberry leaf samples with Hongyan, Ningyu, and Taoxun). The multi-cultivar prediction model is executed as follows: (1) establish three dedicated individual-cultivar prediction models for 
estimating the SPAD values of strawberry leaf samples with different cultivars of Hongyan, Ningyu and Taoxun respectively; (2) recognize and identify the cultivar of the unknown leaf samples using the cultivar recognition model; (3) select dedicated individual-cultivar model for SPAD values detection according to result of the cultivar recognition; (4) monitor nitrogen status and guide $\mathrm{N}$ fertilization recommendations on strawberry on the basis of leaf SPAD value.

\section{Model performance assessment}

The prediction performance for cultivar calibration models were assessed by the following indices: root-mean-square errors of the calibration (RMSEC) and prediction (RMSEP), and correlation coefficient of calibration $\left(\mathrm{R}_{\text {cal }}\right)$ and prediction $\left(\mathrm{R}_{\text {pre }}\right)$ [44]. The main evaluation indices for cultivar calibration models are the $\mathrm{R}_{\text {pre }}$ and RMSEP values. These indices are defined in the following equations:

$$
R_{\text {cal }}=\sqrt{\frac{\sum_{i=1}^{n_{\text {cal }}}\left(y_{p i}-y_{m i}\right)^{2}}{\sum_{i=1}^{n_{c a l}}\left(y_{p i}-y_{\text {mean }}\right)^{2}}}
$$

$$
R_{\mathrm{pre}}=\sqrt{\frac{\sum_{i=1}^{n_{\text {pre }}}\left(y_{p i}-y_{m i}\right)^{2}}{\sum_{i=1}^{n_{\text {pre }}}\left(y_{p i}-y_{\text {mean }}\right)^{2}}}
$$

$$
\text { RMSEC }=\sqrt{\frac{1}{n_{c a l}} \sum_{i=1}^{n_{c a l}}\left(y_{p i}-y_{m i}\right)^{2}}
$$

$$
R M S E P=\sqrt{\frac{1}{n_{\mathrm{pre}}} \sum_{i=1}^{n_{\text {pre }}}\left(y_{p i}-y_{m i}\right)^{2}}
$$

where $y_{m i}$ is the measured SPAD value in leaf sample number $\mathrm{i} ; y_{p i}$ is the predicted SPAD value in leaf sample number $i ; y_{\text {mean }}$ is the average SPAD value in the prediction or calibration set; $n_{\text {pre }}$ and $n_{c a l}$ are numbers of leaves samples in the 
test and training set, separately. Generally, an ideal model should exhibit higher $R_{\text {pre }}$ value and lower RMSEP value.

\section{Results and discussion}

\section{Spectral behaviors of multi-cultivar samples}

The reflectance spectrum demonstrated in Fig. 1 appeared typical spectral characteristics of strawberry leaves with three different cultivars. The responses of the spectrum to chlorophyll and nitrogen content were similar in trend and different in corresponding reflectivity. This variation might be related to the difference in cellular structure and optical propagation properties of strawberry leaves with different cultivars. It could be observed that the wave peak appeared around the wavelength of $820 \mathrm{~nm}$ and wave valley appeared around the wavelength of $650 \sim 700 \mathrm{~nm}$ in the spectral curve. The former was related with the strong reflection of chlorophyll, while the latter was ascribed to strong absorption of chlorophyll [45]. Moreover, the spectral reflectance increased rapidly at NIR region $(700 \sim 750 \mathrm{~nm})$, which verified the feasibility of using NIR location to predict chlorophyll concentration and $\mathrm{N}$ content in strawberry leaves.

\section{Fig. 2 Spectral behaviors of strawberry leaves with different cultivars}

\section{Statistics for measurement of SPAD values}

The range, mean and standard deviation (S.D.) of SPAD value of leaf samples with different cultivars are depicted in Table 1 . There were significant differences in leaf SPAD values among the three strawberry cultivars, indicating that the difference between cultivars could not be reduced or eliminated when individual-cultivar SPAD 
value is applied for assessing chlorophyll concentration and nitrogen status in strawberry leaves with multiple cultivars. Therefore, the different threshold SPAD value has to be established for each cultivar in $\mathrm{N}$ fertilizer management. For the leaf samples with each cultivar, there is a relatively large SPAD value change scope, which is beneficial for developing a calibration model with good performance.

\section{Table 1 Statistics of SPAD values in strawberry leaf samples with three cultivars}

\section{Characteristic fingerprint spectrum exploration}

In order to reduce spatial dimension of the data set and magnify the spectral pattern differences between strawberry leaves with different cultivars, characteristic fingerprint spectrum extraction was carried out in the data space using Random Frog algorithm. Fig. 3 (A) shows that the distribution and selection probability of each variable determined by the Random Frog algorithm. The effectiveness and specificity of each wavelength in cultivar recognition can be determined by evaluating the selection probability of wavelength. Only those wavelengths whose selection probability exceeds the cutoff threshold (0.15) were identified as characteristic fingerprint spectrum, while the others were eliminated. Ultimately, eight characteristic fingerprint spectral bonds $(593.14,561.54,595.61,560.83,575.78,562.26,554.76$, and $569.74 \mathrm{~cm}^{-1}$ ) that marked by red circles in Fig. 3 (A) were extracted and applied for development of cultivar classification model.

Fig. 3. Spectral data optimization and analysis

\section{A. Characteristic fingerprint spectrum extraction;}




\section{Results of cultivar recognition using Random forest}

The Random forest classifier was utilized to establish the classification model for strawberry leaves recognition with multiple cultivars (Hongyan, Ningyu and Taoxun) using the extracted characteristic fingerprint spectrum as inputs. In the model, the fingerprint spectral data were used as $\mathrm{X}$ variables and the $\mathrm{Y}$ variables were related to the class labels of strawberry cultivars. Three cultivars of leaf samples were labeled, where value 1, 2, and 3 were assigned as Hongyan, Ningyu and Taoxun and their corresponding individual-cultivar prediction models respectively. Furthermore, the generalization performance of random forest is affected by the number of the decision trees. In order to decrease the influence of randomness, the following processing is performed: 100 random forest models are established after the number of decision tree is determined, and the average of their accuracy is taken as the classification accuracy under the number of current decision tree. The results of classification accuracy by the Random Forest classifier using different number of decision tree were presented in Fig. 4 (A). As can be seen in Fig. 4 (A), it is ideal to select 300 trees in the random forest in this study by comprehensively considering the number of decision trees and the speed of modeling. The overall classification accuracy of the optimal Random forest model for cultivar recognition on all studied strawberry leaf samples was $98.89 \%$ (Fig. 4 (B)), which indicated that the corresponding individual-cultivar prediction model could be searched accurately based on the excellent result acquired by the cultivar recognition model through the model search strategy. Therefore, the cultivar recognition model was identified to be beneficial for subsequent multi-cultivar model 
development and improvement due to its high accuracy and reliability, and thus improved $\mathrm{N}$ fertilizer managements for different cultivars of strawberries in precision agriculture.

\section{Fig. 4. Performance of cultivar recognition model developed by Random Forest.}

\section{(A) The generalization performance of Random Forest}

(B) The result of cultivar recognition model

\section{Optimization of cultivar models}

To eliminate irrelevant spectral information and meet the real time application requirements, it is important to extract optimal wavelengths using SPA algorithm for optimizing SPAD values detection models, which may be more meaningful than that using full wavelength for estimating the nitrogen status in strawberry and improving $\mathrm{N}$ fertilizer managements in precision agriculture. The distribution and number of optimal wavelengths for individual-cultivar models, hybrid-cultivar model and multi-cultivar model were respectively shown in Fig. 3 (B) and Table 2.

\section{Comparison analysis of individual-cultivar models and hybrid-cultivar model in} SPAD values prediction

The performances of individual-cultivar models respectively developed according to the single cultivar of strawberry leaf including Hongyan, Ningyu and Taoxun in SPAD values prediction were illustrated in Table 2. In order to evaluate the influence of cultivar variation, individual-cultivar models were verified through different independent prediction sets and other cultivar datasets. It was found that individual-cultivar models demonstrated satisfactory prediction performance if the 
372

predicted samples belonged to the same strawberry cultivar as the calibration samples. However, when applied to measure SPAD values of leaf samples from the other strawberry cultivars, the performance and universalities of dedicated individual-cultivar model decreased significantly due to difference in cellular structure and optical propagation properties between different cultivars of strawberry leaves. For instance, as shown in Fig. 5 (A), when the individual-cultivar model of Ningyu was utilized to predict the SPAD values of samples from other cultivars of Hongyan and Taoxun, the RMSEP and $R_{p}$ values were merely $3.617,2.529$ and $0.147,0.243$, respectively. The poor overall performance was also shown in other two individual-cultivar models and incompleted hybrid-cultivar models (Fig. 5 (B)), which indicated that when determined SPAD values of leaf samples with multiple strawberry cultivars, the individual-cultivar prediction model was hypersensitive to the cultivars variation due to variability of cellular structural differences among the leaves that cause different light scattering and/or reflection effects.

In an attempt to correct for the influence of variation of strawberry cultivars in SPAD value prediction, the hybrid-cultivar prediction model was established based on calibration set containing strawberry leaf samples with three diverse cultivars. The performance of the hybrid-cultivar model is indicated in Fig. 5 (C) and Table 2. Compared to the individual-cultivar models, although the result of the hybrid-cultivar model was a little worse than those from dedicated individual-cultivar models for prediction on the same cultivars of samples, the hybrid-cultivar model was considered more robust and reliable because it generated better results for all the samples of 
394

395

diverse cultivars, with the overall $R_{p}$ and RMSEP values of 0.901 and 0.687 , respectively. The result denoted that hybrid-model decreased the impact of cultivar variation on the robustness and stability for SPAD values measurement and was not sensitive to the cultivar variation to some extent. However, the resistance of hybrid-model to cultivar variation would decrease as samples increase, and thus the prediction accuracy and reliability drop gradually. On the other hand, when future strawberry leaf samples with new cultivars are used in the calibration set, the previous hybrid-cultivar would not be applicable and require be rebuilding and updating. In conclusion, individual-cultivar models and hybrid-cultivar model were not reliable enough for leaf chlorophyll content and nitrogen status estimation on strawberry in $\mathrm{N}$ fertilizer managements.

\section{Table 2-Performance of different cultivar models in SPAD values prediction}

\section{Analysis of multi-cultivar model on SPAD values prediction}

Considering the different defects of individual-cultivar model and hybrid-cultivar model, the multi-cultivar model that combined the result of cultivar recognition, individual-cultivar models, and model search strategy was proposed and developed. The performance of the multi-cultivar model for predicting SPAD values of strawberry leaves with three varying cultivar is also illustrated in Table 2 and Fig. 5 (D). Compared to individual-cultivar models and hybrid-cultivar model, the multi-cultivar model shown better accuracy and performance, with the overall $R_{p}$ and RMSEP values of 0.966 and 0.468 respectively. According to the principle of multi-cultivar model establishment, we could conclude that the prediction accuracy of multi-cultivar model would be infinitely close to the individual-cultivar model that 
applied to the prediction of the leaf sample from same strawberry cultivar when the correct recognition rate of cultivar classification model approached $100 \%$. Hence, the multi-cultivar model could render the cultivar variation to negligible part in SPAD values detection. Furthermore, it could be updated and expanded easily when new strawberry cultivars required to be considered, and we just need to establish a new individual-cultivar model of new future samples for the SPAD values prediction. Therefore, the multi-cultivar model was perfectly suited for estimating chlorophyll content and nitrogen status in leaf, and thus beneficial to real-time monitored the different $\mathrm{N}$ requirement of strawberry with diverse cultivars and improved $\mathrm{N}$ fertilizer managements effectively.

Fig. 5. Performance of diverse cultivar models for SPAD values prediction.

\section{Conclusions}

The influence of cultivar variation on the FT-NIR spectral analysis of SPAD values and $\mathrm{N}$ status in strawberry leaves was investigated. A total of 300 strawberry leaves samples from three different cultivars (hundred samples * three cultivars) were used for NIR analysis at $340-1030 \mathrm{~cm}^{-1}$, and chemometrics of PLS was employed for regression analysis. In order to decrease the effect of cultivar variability, individual-cultivar, hybrid-cultivar and multi-cultivar models were respectively constructed with optimal wavelengths as inputs and predictive capacity of models were investigated and compared detailedly. The results of comparison analysis indicated that the multi-cultivar model generated better precision and robustness in predicting leaf SPAD values of strawberry from diverse cultivars, with the overall Rp 
and RMSEP value respectively being 0.966 and 0.468 , which reflected multi-cultivar model was insensitive to cultivar variation and could render it to negligible part in SPAD values detection. It could be concluded that the multi-cultivar model is a promising method to analyze the difference of the dynamic $\mathrm{N}$ requirement in different cultivars of strawberries, real-time monitor the strawberry growth status and then guide the $\mathrm{N}$ fertilization managements. Future studies will focus on further optimizing the performance of the multi-cultivar prediction model, and broadening its application to the detection of various chemical properties in other fruit and plants.

\section{Acknowledgments}

We would like to thank Mrs. Wei Zheng and Xi Yang for the assistance with data collection and spectrum analyses.

\section{Authors' contributions}

In this paper, GW was responsible for part of the test method query and hardware construction, manuscript writing. $\mathrm{BZ}$ was responsible for the implementation of the experiment and revision of manuscript; QJ was responsible for the collection of leaves samples and the acquisition of spectral data and SPAD values of strawberry leaves; YB was responsible for the analysis and verification of data and results, and contributed in writing the manuscript; CT and WP helped design the experiments and co-supervised the study. All authors read and approved the final manuscript.

\section{Funding}

This work was financially supported by Science and Technology projects plan of Jiangsu Vocational College of Agriculture and Forestry (Grant No. 2018kj11 and 
2018kj12).

\section{Availability of data and materials}

The datasets in this study are available from the corresponding author on reasonable request.

\section{Ethics approval and consent to participate}

Not applicable.

\section{Consent for publication}

All authors agreed to publish this manuscript.

\section{Competing interests}

The authors declare that they have no competing interests.

\section{Author details}

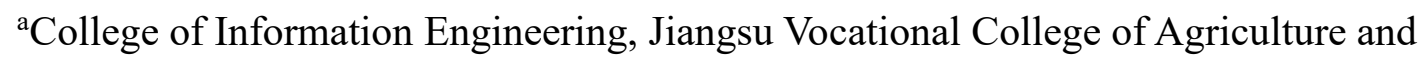
Forestry, Jurong, Jiangsu, PR China

${ }^{\mathrm{b} C o l l e g e}$ of Engineering, Nanjing Agricultural University, Nanjing, Jiangsu, PR China

\section{References}

1. Shao, Y., Zhao, C., Bao, Y., \& He, Y. (2012). Quantification of nitrogen status in rice by least squares support vector machines and reflectance spectroscopy. Food and Bioprocess Technology, 5(1), 100-107.

2. Broge, N. H., \& Leblanc, E. (2001). Comparing prediction power and stability of broadband and hyperspectral vegetation indices for estimation of green leaf area index and canopy chlorophyll density. Remote sensing of environment, 76(2), 156-172.

3. Bojović, B., \& Marković, A. (2009). Correlation between nitrogen and chlorophyll content in wheat (Triticum aestivum L.). Kragujevac Journal of Science, 31, 69-74. 
4. Neufeld, H. S., Chappelka, A. H., Somers, G. L., Burkey, K. O., Davison, A. W., \& Finkelstein, P. L. (2006). Visible foliar injury caused by ozone alters the relationship between SPAD meter readings and chlorophyll concentrations in cutleaf coneflower. Photosynthesis Research, 87(3), 281-286.

5. Minolta, C. (1989). Manual for chlorophyll meter SPAD-502. Osaka: Minolta Radiometric Instruments Divisions.

6. Hu, M., Zhai, G., Zhao, Y., \& Wang, Z. (2018). Uses of selection strategies in both spectral and sample spaces for classifying hard and soft blueberry using near infrared data. Scientific reports, 8(1), 6671.

7. Feng, W., Yao, X., Zhu, Y., Tian, Y. C., \& Cao, W. X. (2008). Monitoring leaf nitrogen status with hyperspectral reflectance in wheat. European Journal of Agronomy, 28(3), 394-404.

8. Min, M., Lee, W. S., Kim, Y. H., \& Bucklin, R. A. (2006). Nondestructive detection of nitrogen in Chinese cabbage leaves using VIS-NIR spectroscopy. HortScience, 41(1), 162-166.

9. Belton, P. S., Colquhoun, I. J., Kemsley, E. K., Delgadillo, I., Roma, P., Dennis, M. J., ... \& Spraul, M. (1998). Application of chemometrics to the 1H NMR spectra of apple juices: discrimination between apple varieties. Food Chemistry, 61(1-2), 207-213.

10. De Luca, M., Terouzi, W., Kzaiber, F., Ioele, G., Oussama, A., \& Ragno, G. (2012). Classification of moroccan olive cultivars by linear discriminant analysis applied to ATR-FTIR spectra of endocarps. International journal of food science \& technology, 47(6), 1286-1292.

11. Gouvinhas, I., de Almeida, J. M., Carvalho, T., Machado, N., \& Barros, A. I. (2015). Discrimination and characterisation of extra virgin olive oils from three cultivars in different maturation stages using Fourier transform infrared spectroscopy in tandem with chemometrics. Food chemistry, 174, 226-232.

12. Fan, S., Li, J., Xia, Y., Tian, X., Guo, Z., \& Huang, W. (2019). Long-term evaluation of soluble solids content of apples with biological variability by using near-infrared spectroscopy and calibration transfer method. Postharvest Biology 
and Technology, 151, 79-87.

13. Weng, S., Zhu, W., Zhang, X., Yuan, H., Zheng, L., Zhao, J., ... \& Han, P. (2019). Recent advances in Raman technology with applications in agriculture, food and biosystems: A review. Artificial Intelligence in Agriculture.

14. Güler, S., Macit, I., Koc, A., \& Ibrikci, H. (2006). Estimating leaf nitrogen status of strawberry by using chlorophyll meter reading. Journal of Biological Sciences, 6(6), 1011-1016.

15. Ghaderi, N., \& Siosemardeh, A. (2011). Response to drought stress of two strawberry cultivars (cv. Kurdistan and Selva). Horticulture, Environment, and Biotechnology, 52(1), 6-12.

16. Zhang, B., Dai, D., Huang, J., Zhou, J., Gui, Q., \& Dai, F. (2018). Influence of physical and biological variability and solution methods in fruit and vegetable quality nondestructive inspection by using imaging and near-infrared spectroscopy techniques: A review. Critical reviews in food science and nutrition, 58(12), 2099-2118.

17. Bavec, F., \& Bavec, M. (2001). Chlorophyll meter readings of winter wheat cultivars and grain yield prediction. Communications in soil science and plant analysis, 32(17-18), 2709-2719.

18. Xiong, D., Chen, J., Yu, T., Gao, W., Ling, X., Li, Y., ... \& Huang, J. (2015). SPAD-based leaf nitrogen estimation is impacted by environmental factors and crop leaf characteristics. Scientific reports, 5, 13389.

19. Azia, F., \& Stewart, K. A. (2001). Relationships between extractable chlorophyll and SPAD values in muskmelon leaves. Journal of Plant Nutrition, 24(6), 961-966.

20. Xu, L., Li, J., \& Zhang, D. (2018). Near-infrared light penetration depth analysis inside melon with thick peel by a novel strategy of slicing combining with least square fitting method. Journal of food process engineering, 41(7), e12886.

21. Li, J., Zhang, H., Zhan, B., Wang, Z., \& Jiang, Y. (2019). Determination of SSC in pears by establishing the multi-cultivar models based on visible-NIR spectroscopy. Infrared Physics \& Technology, 102, 103066. 
22. Wang, J., Wang, J., Chen, Z., \& Han, D. (2017). Development of multi-cultivar models for predicting the soluble solid content and firmness of European pear (Pyrus communis L.) using portable vis-NIR spectroscopy. Postharvest biology and technology, 129, 143-151.

23. Li, X., Wang, S., Shi, W., \& Shen, Q. (2016). Partial least squares discriminant analysis model based on variable selection applied to identify the adulterated olive oil. Food Analytical Methods, 9(6), 1713-1718.

24. Chen, X., Ding, Q., He, R., \& Li, H. (2019). A protocol of field-based phenotyping procedure for no-till wheat root system architecture based on data-driven model-assist. Artificial Intelligence in Agriculture.

25. Imanishi, J., Nakayama, A., Suzuki, Y., Imanishi, A., Ueda, N., Morimoto, Y., \& Yoneda, M. (2010). Nondestructive determination of leaf chlorophyll content in two flowering cherries using reflectance and absorptance spectra. Landscape and ecological engineering, 6(2), 219-234.

26. Saptoro, A., Tadé, M. O., \& Vuthaluru, H. (2012). A modified Kennard-Stone algorithm for optimal division of data for developing artificial neural network models. Chemical Product and Process Modeling, 7(1).

27. Wang, H. F., Huo, Z. G., Zhou, G. S., Liao, Q. H., Feng, H. K., \& Wu, L. (2016). Estimating leaf SPAD values of freeze-damaged winter wheat using continuous wavelet analysis. Plant physiology and biochemistry, 98, 39-45.

28. Li, S., Luo, H., Hu, M., Zhang, M., Feng, J., Liu, Y., ... \& Liu, B. (2019). Optical non-destructive techniques for small berry fruits: a review. Artificial Intelligence in Agriculture.

29. Savitzky, A., \& Golay, M. J. (1964). Smoothing and differentiation of data by simplified least squares procedures. Analytical chemistry, 36(8), 1627-1639.

30. Iñón, F. A., Garrigues, J. M., Garrigues, S., Molina, A., \& de la Guardia, M. (2003). Selection of calibration set samples in determination of olive oil acidity by partial least squares-attenuated total reflectance-Fourier transform infrared spectroscopy. Analytica Chimica Acta, 489(1), 59-75.

31. Çarçabal, P., Kroemer, R. T., Snoek, L. C., Simons, J. P., Bakker, J. M., 
Compagnon, I., ... \& von Helden, G. (2004). Hydrated complexes of tryptophan: ion dip infrared spectroscopy in the 'molecular fingerprint'region, 100-2000 cm1. Physical Chemistry Chemical Physics, 6(19), 4546-4552.

32. Okamoto, H. (2000). Picosecond transient infrared spectrum of 4-(dimethylamino) benzonitrile in the fingerprint region. The Journal of Physical Chemistry A, 104(18), 4182-4187.

33. Zhang, L., Zhang, B., Zhou, J., Gu, B., \& Tian, G. (2017). Uninformative Biological Variability Elimination in Apple Soluble Solids Content Inspection by Using Fourier Transform Near-Infrared Spectroscopy Combined with Multivariate Analysis and Wavelength Selection Algorithm. Journal of analytical methods in chemistry, 2017.

34. Li, H. D., Xu, Q. S., \& Liang, Y. Z. (2012). Random frog: an efficient reversible jump Markov Chain Monte Carlo-like approach for variable selection with applications to gene selection and disease classification. Analytica Chimica Acta, 740, 20-26.

35. Yun, Y. H., Li, H. D., Wood, L. R., Fan, W., Wang, J. J., Cao, D. S., ... \& Liang, Y. Z. (2013). An efficient method of wavelength interval selection based on random frog for multivariate spectral calibration. Spectrochimica Acta Part A: Molecular and Biomolecular Spectroscopy, 111, 31-36.

36. Hu, M. H., Dong, Q. L., Liu, B. L., Opara, U. L., \& Chen, L. (2015). Estimating blueberry mechanical properties based on random frog selected hyperspectral data. Postharvest Biology and Technology, 106, 1-10.

37. Bai, Y., Xiong, Y., Huang, J., Zhou, J., \& Zhang, B. (2019). Accurate prediction of soluble solid content of apples from multiple geographical regions by combining deep learning with spectral fingerprint features. Postharvest Biology and Technology, 156, 110943.

38. Breiman, Random forests, Mach. Learn. 45 (1) (2001) 5-32

39. Mursalin, M., Zhang, Y., Chen, Y., \& Chawla, N. V. (2017). Automated epileptic seizure detection using improved correlation-based feature selection with random forest classifier. Neurocomputing, 241, 204-214. 
40. Huang, H., Yu, H., Xu, H., \& Ying, Y. (2008). Near infrared spectroscopy for on/in-line monitoring of quality in foods and beverages: A review. Journal of food engineering, 87(3), 303-313.

41. Zhu, H., Chu, B., Zhang, C., Liu, F., Jiang, L., \& He, Y. (2017). Hyperspectral imaging for presymptomatic detection of tobacco disease with successive projections algorithm and machine-learning classifiers. Scientific reports, 7(1), 4125.

42. Cheng, J. H., Sun, D. W., \& Pu, H. (2016). Combining the genetic algorithm and successive projection algorithm for the selection of feature wavelengths to evaluate exudative characteristics in frozen-thawed fish muscle. Food chemistry, $197,855-863$.

43. Galvao, R. K. H., Araujo, M. C. U., Fragoso, W. D., Silva, E. C., Jose, G. E., Soares, S. F. C., \& Paiva, H. M. (2008). A variable elimination method to improve the parsimony of MLR models using the successive projections algorithm. Chemometrics and intelligent laboratory systems, 92(1), 83-91.

44. Lyu, Q., Liao, Q., Liu, Y., \& Lan, Y. (2015). Feasibility of SSC prediction for navel orange based on origin recognition using NIR spectroscopy. Intelligent Automation \& Soft Computing, 21(3), 305-317.

45. Zheng, T., Liu, N., Wu, L., Li, M., Sun, H., Zhang, Q., \& Wu, J. (2018). Estimation of Chlorophyll Content in Potato Leaves Based on Spectral Red Edge Position. IFAC-PapersOnLine, 51(17), 602-606. 


\section{Figure captions:}

630 Figure. 1. Flowchart for prediction of SPAD values of strawberry leaves with different cultivars using multi-cultivar model.

632 Figure. 2. Spectral behaviors of strawberry leaves with different cultivars

633 Figure. 3. Spectral data optimization and analysis

634

635

636

637

638

639

640

641
A. Characteristic fingerprint spectrum extraction;

B. Distribution of optimal wavelengths selected by SPA on the full spectrum.

Figure. 4. Performance of cultivar recognition model developed by Random Forest.

A. The generalization performance of Random Forest

B. The result of cultivar recognition model

Figure. 5. Performance of diverse cultivar models for SPAD values prediction. 


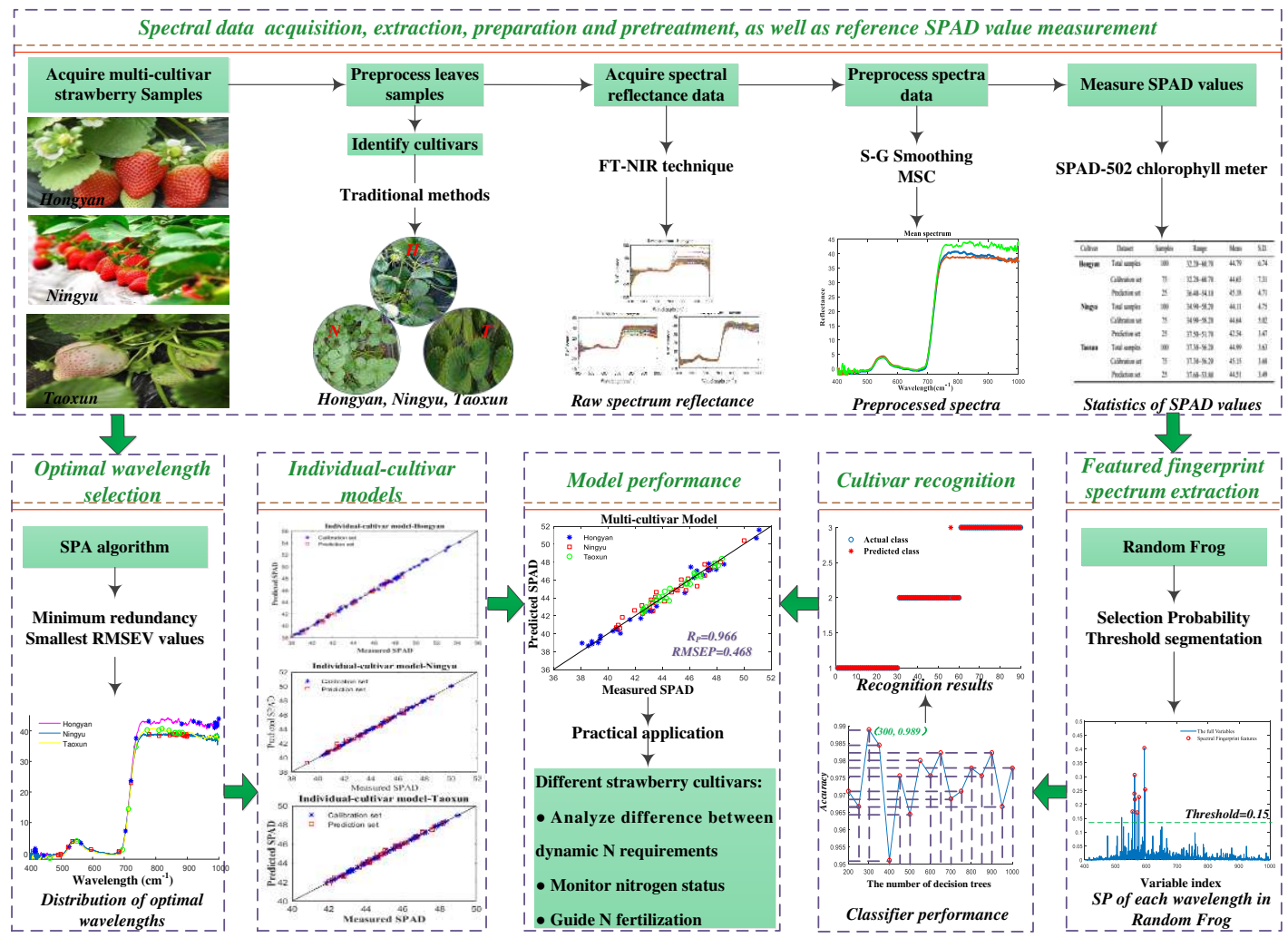

643 Figure. 1. Flowchart for prediction of SPAD values of strawberry leaves with different 


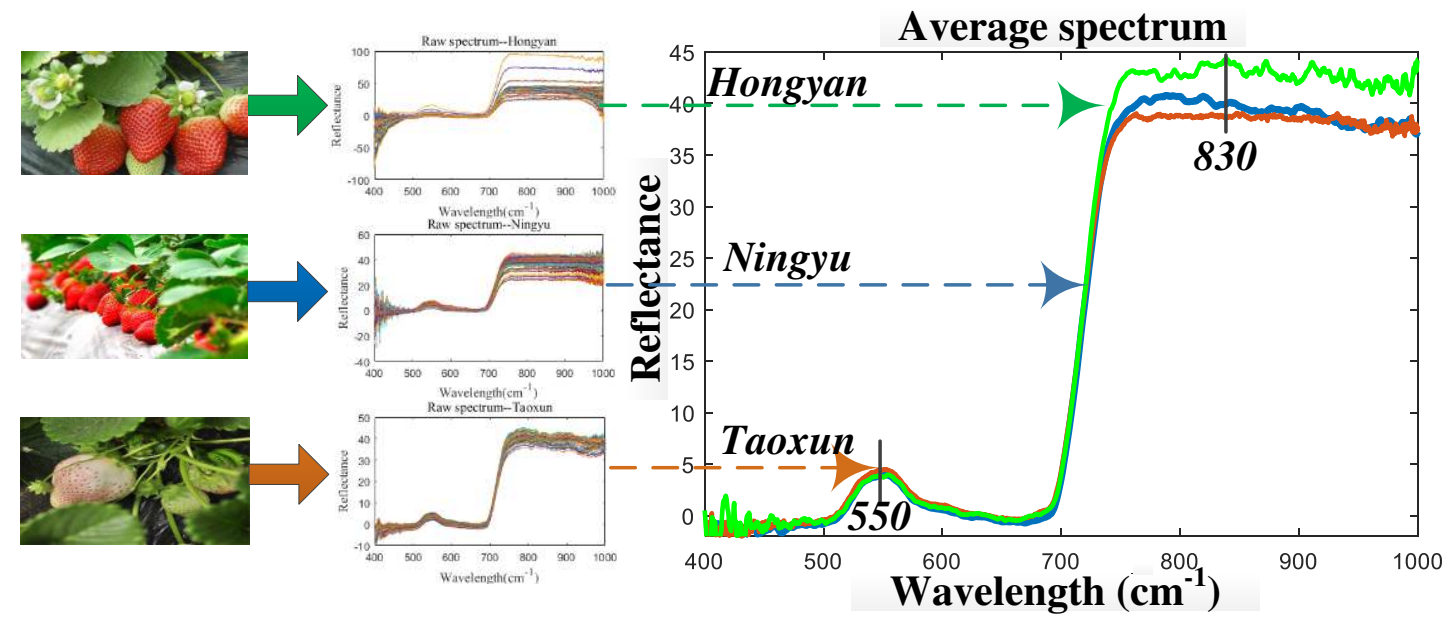

647

Fig. 2 Spectral behaviors of strawberry leaves with different cultivars

648

649

650

651 


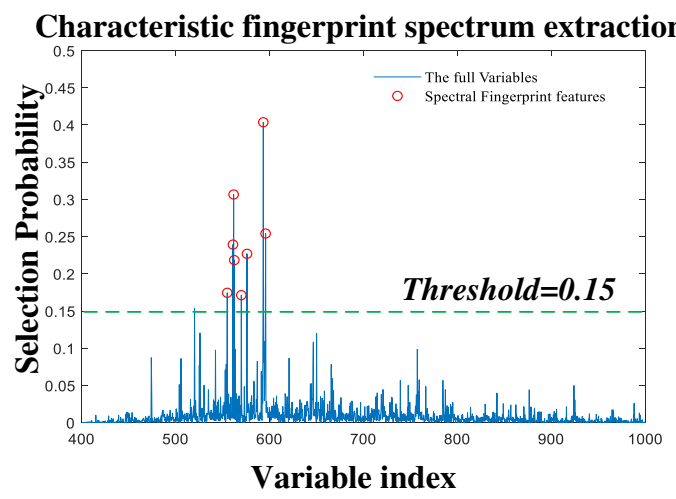

(A)

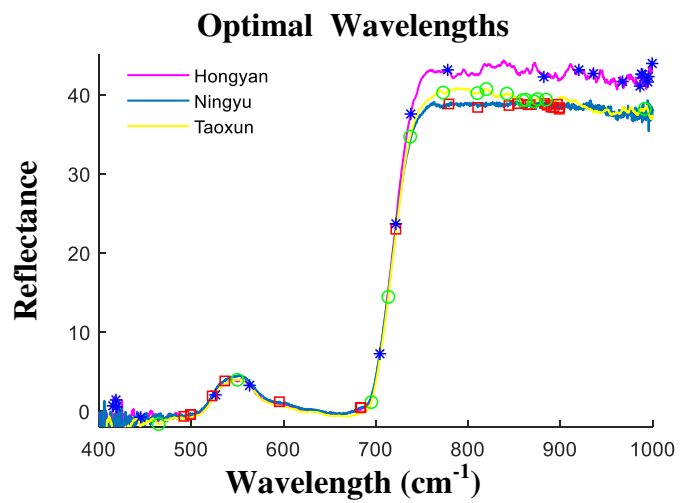

(B)
652

653

654

655

656

657

658

Fig. 3. Spectral data optimization and analysis

A. Characteristic fingerprint spectrum extraction;

B. Distribution of optimal wavelengths selected by SPA on the full spectrum. 


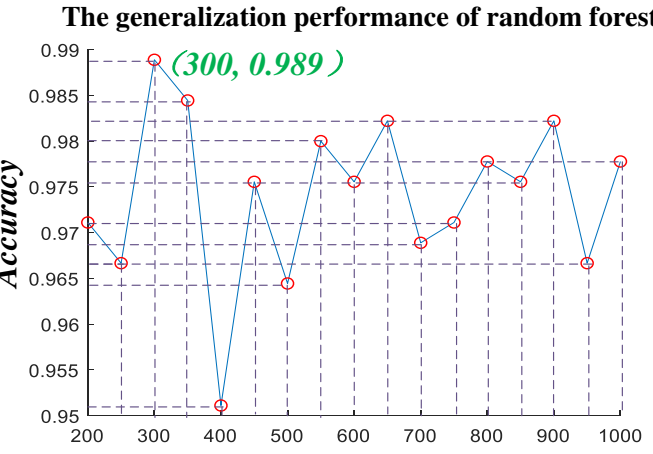

The number of decision trees

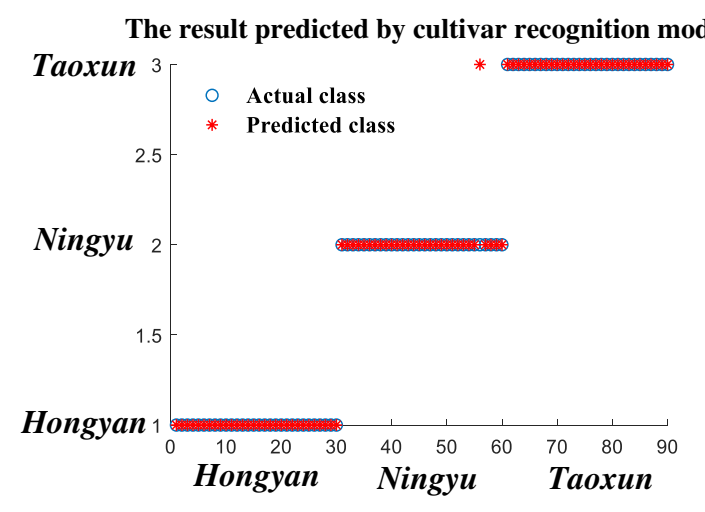

(B)

660 Fig. 4. Performance of cultivar recognition model developed by Random Forest.

(A) The generalization performance of Random Forest 


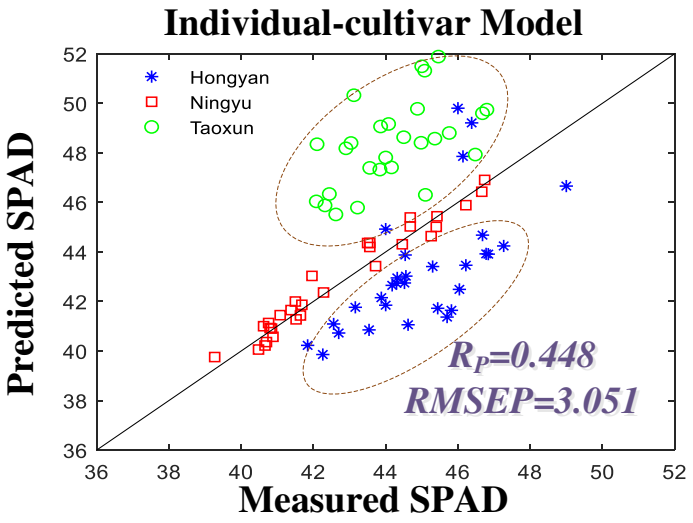

(A)

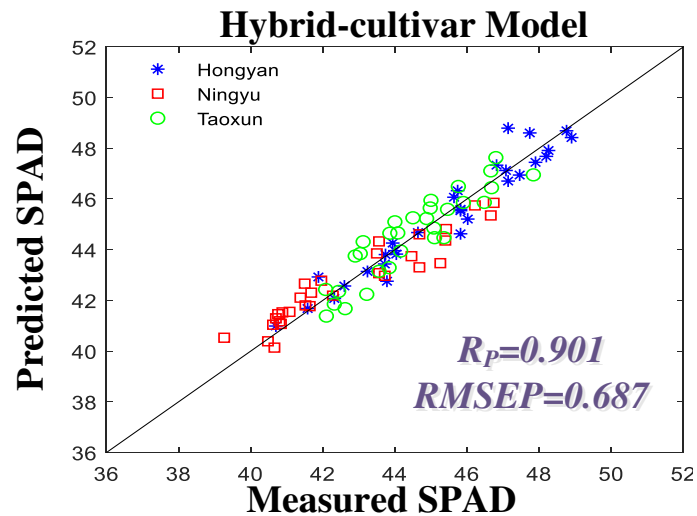

(C)

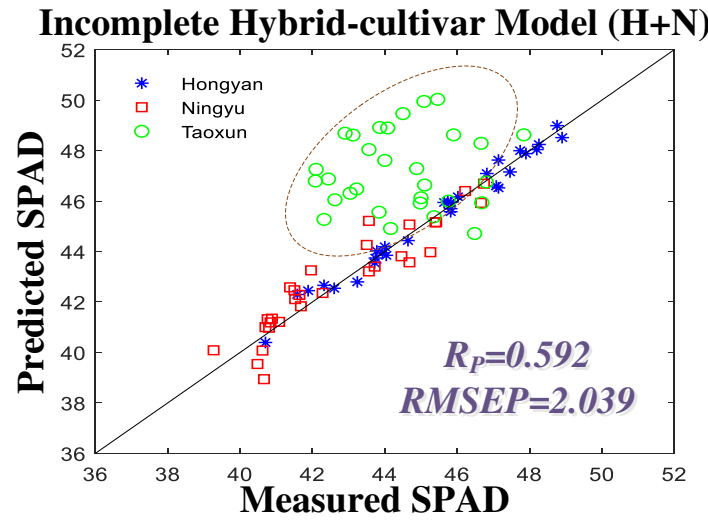

(B)

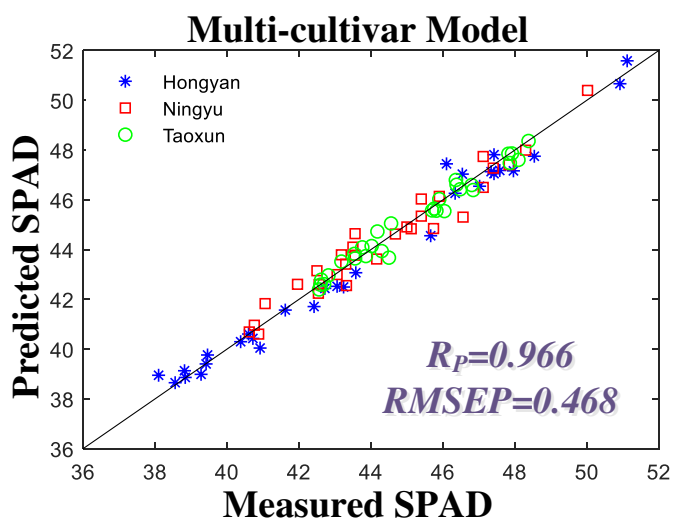

(D) 
668 Table 1-Statistics of SPAD values in strawberry leaf samples with three cultivars

\begin{tabular}{cccccc}
\hline Cultivar & Dataset & Samples & Range & Mean & S.D. \\
\hline Hongyan & Total samples & 100 & $32.20-60.70$ & 44.79 & 6.74 \\
& Calibration set & 75 & $32.20-60.70$ & 44.65 & 7.31 \\
& Prediction set & 25 & $36.40-54.10$ & 45.18 & 4.71 \\
\multirow{5}{*}{ Ningyu } & Total samples & 100 & $34.90-58.20$ & 44.11 & 4.75 \\
& Calibration set & 75 & $34.90-58.20$ & 44.64 & 5.02 \\
& Prediction set & 25 & $37.50-51.70$ & 42.54 & 3.47 \\
& Total samples & 100 & $37.30-56.20$ & 44.99 & 3.63 \\
& Calibration set & 75 & $37.30-56.20$ & 45.15 & 3.68 \\
& Prediction set & 25 & $37.60-53.80$ & 44.51 & 3.49 \\
\hline
\end{tabular}

669

670 


\begin{tabular}{|c|c|c|c|c|c|c|c|c|c|}
\hline \multirow{3}{*}{ Cultivar Model } & \multirow{3}{*}{ EWs } & \multicolumn{6}{|c|}{ Individual sets } & & \\
\hline & & \multicolumn{2}{|c|}{ Hongyan } & \multicolumn{2}{|c|}{ Ningyu } & \multicolumn{2}{|c|}{ Taoxun } & \multicolumn{2}{|c|}{ Overall set } \\
\hline & & RMSEP & $R_{p}$ & RMSEP & $R_{p}$ & RMSEP & $R_{p}$ & RMSEP & $R_{p}$ \\
\hline $\begin{array}{c}\text { Individual-cultivar model } \\
\text { (Hongyan) }\end{array}$ & 20 & 0.551 & 0.979 & 1.473 & 0.511 & 1.378 & 0.617 & 1.642 & 0.519 \\
\hline $\begin{array}{l}\text { Individual-cultivar model } \\
\text { (Ningyu) }\end{array}$ & 22 & 3.617 & 0.147 & 0.536 & 0.949 & 2.529 & 0.243 & 3.051 & 0.448 \\
\hline $\begin{array}{c}\text { Individual-cultivar model } \\
\text { (Taoxun) }\end{array}$ & 14 & 2.087 & 0.213 & 1.851 & 0.227 & 0.317 & 0.971 & 1.604 & 0.348 \\
\hline $\begin{array}{l}\text { Hybrid-cultivar model } \\
\qquad(\mathrm{Hy}+\mathrm{Ny}+\mathrm{Tx})\end{array}$ & 32 & 0.586 & 0.933 & 0.776 & 0.864 & 0.686 & 0.801 & 0.687 & 0.901 \\
\hline Multi-cultivar model & $20 / 22 / 14$ & 0.551 & 0.979 & 0.536 & 0.949 & 0.317 & 0.971 & 0.468 & 0.966 \\
\hline
\end{tabular}

